\title{
Droplet activation parameterization: the population-splitting concept revisited
}

\author{
R. Morales Betancourt ${ }^{1}$ and A. Nenes ${ }^{1,2}$ \\ ${ }^{1}$ School of Earth and Atmospheric Sciences, Georgia Institute of Technology, Atlanta, Georgia, USA \\ ${ }^{2}$ School of Chemical and Biomolecular Engineering, Georgia Institute of Technology, Atlanta, Georgia, USA \\ Correspondence to: A. Nenes (athanasios.nenes@gatech.edu)
}

Received: 12 March 2014 - Published in Geosci. Model Dev. Discuss.: 5 May 2014

Revised: 2 September 2014 - Accepted: 9 September 2014 - Published: 14 October 2014

\begin{abstract}
In this work, we postulate, implement and evaluate modifications to the "population-splitting" concept, introduced by Nenes and Seinfeld (2003), for calculation of water-condensation rates in droplet-activation parameterizations. The population-splitting approximation consists of dividing the population of growing droplets into two categories: those that experience significant growth after exposed to a supersaturation larger than their critical supersaturation, and those that do not grow much larger than their critical diameter. The modifications introduced here lead to an improved accuracy and precision of the parameterizationderived maximum supersaturation, $s_{\max }$, and droplet-number concentration, $N_{\mathrm{d}}$, as determined by comparing against those of detailed numerical simulations of the activation process. A numerical computation of the first-order derivatives $\partial N_{\mathrm{d}} / \partial \chi_{j}$ of the parameterized $N_{\mathrm{d}}$ to input variables $\chi_{j}$ was performed and compared against the corresponding parcelmodel-derived sensitivities, providing a thorough evaluation of the impacts of the introduced modifications in the parameterization ability to respond to aerosol characteristics. An evaluation of the parameterization computation of $N_{\mathrm{d}}$ and $s_{\max }$ against detailed numerical simulations of the activation process showed a relative error of $-6.0 \% \pm 6.2 \%$ for $s_{\max }$, and $-2.7 \% \pm 4.8 \%$ for $N_{\mathrm{d}}$, which represents a considerable reduction in prediction bias when compared to earlier versions of the parameterization. The proposed modifications require only minor changes for their numerical implementation in existing codes based on the population-splitting concept.
\end{abstract}

\section{Introduction}

During the process of cloud formation, preexisting aerosol particles act as cloud condensation nuclei (CCN), upon which cloud droplets first form and subsequently grow. Changes in either the amount or composition of atmospheric aerosol can alter cloud microphysical and optical properties, indirectly impacting the planetary radiation balance and hydrological cycle. Aerosol-cloud interactions constitute some of the most uncertain aspects of anthropogenic climate-change estimates (Intergovernmental Panel on Climate Change, 2007).

Calculation of droplet number in atmospheric models requires the computation of new droplet formation (i.e., droplet activation), which occurs at subgrid scales and its representation is computationally expensive if done explicitly using numerical parcel models. For this reason, parameterizations of the activation process have been developed. In these formulations, the fraction of atmospheric aerosol that activates into cloud droplets is determined for an air parcel that ascends with an updraft velocity $(w)$. These activation parameterizations use a Lagrangian-parcel-model approach to study the detailed process of water-vapor condensation on the population of growing droplets. A thorough review of activation parameterizations can be found in Ghan et al. (2011). Most of these activation schemes follow the framework proposed by the seminal work of Twomey (1959) which involves two conceptual steps. First, the availability of $\mathrm{CCN}$ is determined as function of supersaturation (e.g., using Köhler's theory or an adsorption-activation theory, together with aerosol-size distribution and chemical composition), and second, by approximately solving the water-vapor balance in the 
ascending cloud parcel to determine the maximum supersaturation, $s_{\max }$, attained in it. After this is done, the number of activated cloud droplets, $N_{\mathrm{d}}$, is equal to the concentration of $\mathrm{CCN}$ with a critical supersaturation, $s_{\mathrm{c}}$, lower than $s_{\max }$. A number of activation parameterizations have been developed using this approach (e.g., Feingold and Heymsfield, 1992; Ghan et al., 1993; Nenes and Seinfeld, 2003; Pinsky et al., 2012), and many have been incorporated into Global Circulation Models (GCMs) and regional models to compute aerosol indirect effects (e.g., Abdul-Razzak and Ghan, 2000; Fountoukis and Nenes, 2005; Ming et al., 2006; Shipway and Abel, 2010).

The central problem that these schemes need to address is the correct estimation of the size of the growing droplets at the time of peak supersaturation. The condensation rate of water vapor onto activated droplets in the parcel is proportional to the integral diameter of the growing droplet population, and it therefore plays an important role in defining $s_{\max }$. This task is particularly challenging for the largest particles in the CCN population. As noted by Chuang et al. (1997), a portion of the CCN population - those with relatively low $s_{\mathrm{c}}$ - are "inertially limited" (Nenes et al., 2001) and their size does not equilibrate instantaneously with the ambient supersaturation. Therefore, the equilibrium assumption is not adequate for computing the sizes for these particles. This limitation would likely affect particles larger than approximately $0.2 \mu \mathrm{m}$ in diameter, therefore impacting the coarse mode, as well as a sizable fraction of accumulation-mode particles.

Even though coarse-mode particles typically contribute a small number of concentration to the $\mathrm{CCN}$ population, they represent an important sink for water vapor, effectively modulating the parcel $s_{\max }$ (e.g., Ghan et al., 1998; Barahona et al., 2010; Morales Betancourt and Nenes, 2014). This means that even modest increases in either the number or the hygroscopicity of these large particles can cause a significant decrease in $s_{\max }$, often leading to lower droplet concentrations (Morales Betancourt and Nenes, 2014). Furthermore, because of the large contribution of accumulation-mode particles to the total $\mathrm{CCN}$ active population, accurately accounting for the water uptake of the inertially limited portion of accumulation-mode $\mathrm{CCN}$ is of great importance in determining $s_{\max }$ and $N_{\mathrm{d}}$.

Within the parameterization framework first proposed by Nenes and Seinfeld (2003), different approaches have been incrementally adopted to improve their ability to capture the supersaturation across a large set of conditions. Fountoukis and Nenes (2005) extended this framework to include the effect of mass transfer limitations in the non-continuum regime through an effective water-vapor-accommodation coefficient. Kumar et al. (2009) introduced changes in the CCN spectra to allow for adsorption activation. Barahona and Nenes (2007) introduced a framework to account for the impact of entrainment and mixing in decreasing the condensation rate on the droplets to subadiabatic levels. The prediction of $N_{\mathrm{d}}$ with the parameterization of Fountoukis and Nenes (2005) is typically within $\pm 20 \%$ when compared to parcel-model simulations for a wide range of aerosol conditions and vertical velocity, and is capable of reproducing observed clouddroplet data (Fountoukis et al., 2007; Meskhidze et al., 2005). However, when the population of inertially limited CCN is large, it tends to slightly overestimate $N_{\mathrm{d}}$ and $s_{\max }$. Barahona et al. (2010) noted this and introduced a novel way of approximating the condensation rate on the large particles to better account for their contribution to depleting the available water vapor. This new approach corrected the overprediction issue of Fountoukis and Nenes (2005) in conditions where there is a significant presence of large CCN. As we show in the present work, the modifications by Barahona et al. (2010) nevertheless overrepresent the condensation rate on large CCN, introducing a slight underestimation of $N_{\mathrm{d}}$ and $s_{\max }$ under specific circumstances.

In this work, we introduce modifications to the "population-splitting" concept regarding the computation of droplet size at activation. We first present a brief account of the concepts leading to the population-splitting approach of Nenes and Seinfeld (2003), and then present the proposed modifications. The augmented parameterization is evaluated by comparing computations of $N_{\mathrm{d}}$ and $s_{\max }$ and their sensitivity to aerosol properties against detailed parcel-model simulations.

\section{General framework of activation parameterizations}

The number concentration of aerosol activated into cloud droplets, $N_{\mathrm{d}}$, is the central quantity to be predicted by activation parameterizations. These parameterizations typically determine the maximum supersaturation $s_{\max }$ developed in an ascending air parcel, and then compute $N_{\mathrm{d}}$ as the subset of CCN with a critical supersaturation, $s_{\mathrm{c}}$, less than $s_{\max }$. The maximum supersaturation is attained when the supersaturation production, due to expansion cooling, is balanced by the water-vapor depletion from condensation. If the parcel is ascending with a constant vertical velocity $w$, its supersaturation tendency can be written as (e.g., Pruppacher and Klett, 1997)

$\frac{\mathrm{d} s}{\mathrm{~d} t}=\alpha w-\gamma\left(\frac{\mathrm{d} q_{1}}{\mathrm{~d} t}\right)$

where $\left(\mathrm{d} q_{1} / \mathrm{d} t\right)$ is the rate of change of liquid water mixing ratio in the parcel, $q_{1}$, and $\alpha$ and $\gamma$ are size independent, slowly varying functions of temperature, which can be considered constant during the activation process (see Appendix A). Since condensation transfers mass to the droplet population, the condensation rate in Eq. (1) can be expressed in terms of the droplet-growth rate. Ignoring the effects of curvature and solutes on the equilibrium vapor pressure of the growing droplets, the condensational growth of a droplet 
with diameter $D_{\mathrm{p}}$ is given by (Nenes and Seinfeld, 2003)

$D_{\mathrm{p}} \frac{\mathrm{d} D_{\mathrm{p}}}{\mathrm{d} t}=G s$,

where $G$ is the mass-transfer coefficient of water to the droplets (Appendix A). Since $q_{1}$ is proportional to the total volume concentration of the droplet population, the condensation rate in Eq. (1) can be expressed in terms of $D_{\mathrm{p}}$ by using the growth rate in Eq. (2):

$$
\frac{\mathrm{d} q_{1}}{\mathrm{~d} t}=\frac{\pi}{2} \frac{\rho_{\mathrm{w}}}{\rho_{\mathrm{a}}} G s \int n\left(d_{\mathrm{p}}\right) D_{\mathrm{p}}\left(d_{\mathrm{p}}, t\right) \mathrm{d} d_{\mathrm{p}},
$$

where $D_{\mathrm{p}}\left(d_{\mathrm{p}}, t\right)$ is the wet diameter at a time $t$ after in-cloud ascent of a droplet growing on an aerosol particle of dry size $d_{\mathrm{p}}$. Equation (3) indicates that the condensation rate is proportional to the integral diameter of the droplet size distribution. Using Köhler theory (e.g., Nenes and Seinfeld, 2003) or an adsorption activation theory (Kumar et al., 2009) to relate the dry size of the aerosol, $d_{\mathrm{p}}$, to $s_{\mathrm{c}}$, the integral in Eq. (3) can be expressed in terms of the critical supersaturation $s_{\mathrm{c}}$. Following Nenes and Seinfeld (2003), the integral diameter (also termed "condensation integral") in $s_{\mathrm{c}}$ space is defined here as

$$
I(0, s) \equiv \int_{0}^{s} n\left(s_{\mathrm{c}}\right) D_{\mathrm{p}}\left(s_{\mathrm{c}}, t\right) \mathrm{d} s_{\mathrm{c}},
$$

where the first and second arguments in $I(a, b)$ represent the lower and upper integration limits respectively. The function $n\left(s_{\mathrm{c}}\right)$ is the size distribution of aerosol particles mapped to the critical supersaruration space. Therefore, $n\left(s_{\mathrm{c}}\right) \mathrm{d} s_{\mathrm{c}}$ is the number of particles with a critical supersaturation between $s_{\mathrm{C}}$ and $s_{\mathrm{c}}+\mathrm{d} s_{\mathrm{c}}$. The maximum supersaturation can be found by setting $\mathrm{d} s / \mathrm{d} t=0$ in Eq. (1). Using Eq. (4), and after some manipulation, the supersaturation equation at the moment of maximum supersaturation can be written as

$s_{\max } I\left(0, s_{\max }\right)=\beta$,

with $\beta=2 \rho_{\mathrm{a}} \alpha w /\left(\pi \rho_{\mathrm{w}} \gamma G\right)$. Equation (5) cannot, in general, be solved analytically. The diameter of the growing droplets at peak supersaturation is necessary to calculate the condensation integral, $I\left(0, s_{\max }\right)$, and still requires a formulation in terms of the dry aerosol-size distribution. The populationsplitting approach (Nenes and Seinfeld, 2003) provides such a framework to approximate the size of the growing droplets, $D_{\mathrm{p}}$, and compute $I\left(0, s_{\max }\right)$ by splitting this integral into the separate contributions from two different populations of droplets. These two populations are identified by their different asymptotic growth regimes. The fundamentals of this approximation are briefly explained below.

\subsection{The population-splitting concept}

A solution to the supersaturation balance Eq. (5) requires expression of the condensation rate, proportional to $I\left(0, s_{\max }\right)$, in terms of the dry aerosol-size distribution and the size of droplets at the time of maximum supersaturation, $t_{\mathrm{m}}$. The population-splitting concept is a method to compute the integral $I\left(0, s_{\max }\right)$ of Eq. (4) by dividing the CCN spectrum into different categories. These categories are defined by the approximation used to estimate their size at the moment of maximum supersaturation. The first step is to find an appropriate expression to estimate the size $D_{\mathrm{p}}\left(s_{\mathrm{c}}, t_{\mathrm{m}}\right)$ of a single droplet. This is often done by integrating Eq. (2) from the activation time, $\tau_{s_{\mathrm{c}}}$, defined as $s\left(\tau_{s_{\mathrm{c}}}\right)=s_{\mathrm{c}}$, to the time when $s$ reaches a maximum, i.e.,

$D_{\mathrm{p}}^{2}=D_{\mathrm{p}}\left(\tau_{s_{\mathrm{c}}}\right)^{2}+2 G \int_{\tau_{s_{\mathrm{c}}}}^{t_{\mathrm{m}}} s \mathrm{~d} t$.

Two assumptions, each representing asymptotic growth limits, have been often adopted to obtain an approximate expression for $D_{\mathrm{p}}$ in Eq. (6). One such approximation, denoted here as $D_{\mathrm{p}}^{(1)}$, consists of neglecting droplet growth after activation, and that the droplet diameter at $s_{\max }$ is given by the critical wet diameter $D_{\mathrm{pc}}$, i.e., $D_{\mathrm{p}}^{(1)}=D_{\mathrm{p}}\left(\tau_{s_{\mathrm{c}}}\right)=D_{\mathrm{pc}}$ (e.g., Ghan et al., 1993). Using Köhler theory, $D_{\mathrm{pc}}$ (hence, $D_{\mathrm{p}}^{(1)}$ ) can be written as a function of $s_{\mathrm{c}}$ (see Appendix A):

$D_{\mathrm{p}}^{(1)}=\frac{2 A}{3 s_{\mathrm{c}}}$.

Although adequate for the smallest CCN, Eq. (7) overestimates the wet diameter when applied to the largest particles in the $\mathrm{CCN}$ population. Due to their size, droplets growing on aerosol particles with a dry diameter larger than $\sim 0.2 \mu \mathrm{m}$ cannot grow in equilibrium with the ambient supersaturation (Chuang et al., 1997). As a consequence of this inertial limitation (Nenes et al., 2001), these droplets fall far behind their equilibrium diameter as the parcel supersaturation increases, and therefore application of Eq. (7) leads to a large overestimation of their size. This, in turn, leads to overestimating the condensation rate, biasing $s_{\max }$ and $N_{\mathrm{d}}$ low (Ghan et al., 1993).

Another approximation for $D_{\mathrm{p}}$ in Eq. (6), which we will denote here as $D_{\mathrm{p}}^{(2)}$, first introduced by Twomey (1959), considers that particle growth after exposure to their critical supersaturation is the main contributor to particle size. This approach effectively neglects the initial size of the particles when exposed to $s_{\mathrm{c}}, D_{\mathrm{p}}\left(\tau_{s_{\mathrm{c}}}\right)$, and considers only the contribution of the growth term in Eq. (6). Twomey (1959) further proposed a lower bound for the supersaturation integral relating it to $s_{\mathrm{c}}$, namely

$\int_{\tau_{s_{\mathrm{c}}}}^{t_{\mathrm{m}}} s \mathrm{~d} t=\frac{s_{\mathrm{max}}^{2}-s_{\mathrm{c}}^{2}}{2 \alpha w}$.

However, neglecting $D_{\mathrm{p}}\left(\tau_{s_{\mathrm{c}}}\right)$ can cause a large underestimation of $D_{\mathrm{p}}$, and therefore of the surface area for watervapor condensation, particularly for large $\mathrm{CCN}$. When this 
approximation is adopted, the droplet size $D_{\mathrm{p}}\left(s_{\mathrm{c}}, t_{\mathrm{m}}\right)$ can be found by replacing Eq. (8) into Eq. (6), i.e.,

$D_{\mathrm{p}}^{(2)}=\left(\frac{G}{\alpha w}\right)^{1 / 2}\left(s_{\max }^{2}-s_{\mathrm{c}}^{2}\right)^{1 / 2}$.

Subsequent approaches to the problem have acknowledged that, in actuality, both regimes occur within the same CCN population. Abdul-Razzak et al. (1998) identified these regimes based on the proximity of $s_{\mathrm{c}}$ to $s_{\max }$, proposing that, for particles with $s_{\mathrm{c}} \ll s_{\max }$, the growth term was dominant, while for those with $s_{\mathrm{c}} \sim s_{\max }$, the effect of growth was negligible and their size was close to their activation size.

Nenes and Seinfeld (2003) further built on the above concepts and sought to establish specific criteria for splitting the population of $\mathrm{CCN}$ between particles for which the equilibrium assumption, $D_{\mathrm{p}}=D_{\mathrm{p}}^{(1)}$, was adequate, and those for which the droplet growth contributed more significantly to particle size, i.e., $D_{\mathrm{p}}=D_{\mathrm{p}}^{(2)}$. To partition the CCN population between these regimes, Nenes and Seinfeld (2003) determined the values of $s_{\mathrm{c}}$, for which the critical wet diameter $D_{\mathrm{pc}}$ was equal to the growth term after activation, effectively establishing the boundaries between regimes. Solving the resulting equation, i.e., $D_{\mathrm{p}}^{(1)}=D_{\mathrm{p}}^{(2)}$ for $s_{\mathrm{c}}$, two roots were found to satisfy the equality:

$$
\frac{s_{\mathrm{p}}^{ \pm}}{s_{\max }}=\frac{1}{\sqrt{2}}\left[1 \pm\left(1-\frac{\xi_{\mathrm{c}}^{4}}{s_{\max }^{4}}\right)^{1 / 2}\right]^{1 / 2},
$$

where $\xi_{\mathrm{c}}=\left(16 A^{2} \alpha w / 9 G\right)^{1 / 4}$. These roots define two different regions in $s_{\mathrm{c}}$ space (Fig. 1), one for which the growth term is larger than the critical diameter $\left(D_{\mathrm{p}}^{(1)}<D_{\mathrm{p}}^{(2)}\right)$, and one for which $D_{\mathrm{pc}}$ is larger than the growth term $\left(D_{\mathrm{p}}^{(1)}>D_{\mathrm{p}}^{(2)}\right)$. In terms of the discriminant $\Delta=1-\xi_{\mathrm{c}}^{4} / s_{\max }^{4}$ of Nenes and Seinfeld (2003), two clear regimes arise from Eq. (10), one for $s_{\max }>\xi_{\mathrm{c}}$ (equivalent to the condition $\Delta>0$ ), and another for $s_{\max }<\xi_{\mathrm{c}}$ (equivalent to the condition $\Delta<0$ ).

When $s_{\max }>\xi_{\mathrm{c}}$, both roots $s_{\mathrm{p}}^{ \pm}$are real and define the boundaries that split the $\mathrm{CCN}$ into three different populations. For the smallest particles, those with $s_{\max }>s_{\mathrm{c}}>s_{\mathrm{p}}^{+}$, $D_{\mathrm{p}}^{(1)}>D_{\mathrm{p}}^{(2)}$ because the particles do not have enough time to grow. Owing to the inverse relation between $s_{\mathrm{c}}$ and $D_{\mathrm{pc}}$, those particles with $s_{\mathrm{p}}^{-}>s_{\mathrm{c}}$ have such large critical diameters that they cannot be matched by the growth in Eq. (9), and therefore the same inequality holds for them. For the CCN population in between those with $s_{\mathrm{p}}^{+}>s_{\mathrm{c}}>s_{\mathrm{p}}^{-}$, the growth term is larger than $D_{\mathrm{pc}}$. Finally, when $s_{\max }<\xi_{\mathrm{c}}$, Eq. (10) has no real solutions reflecting the fact that, in this region, the critical diameter $D_{\mathrm{p}}^{(1)}$ is always larger than the growth term, $D_{\mathrm{p}}^{(2)}$.

Nenes and Seinfeld (2003) used the clues provided by this classification to define rules for the estimation of $D_{\mathrm{p}}$. For those CCN with $s_{\max }>s_{\mathrm{c}}>s_{\mathrm{p}}^{+}$(termed here as "population
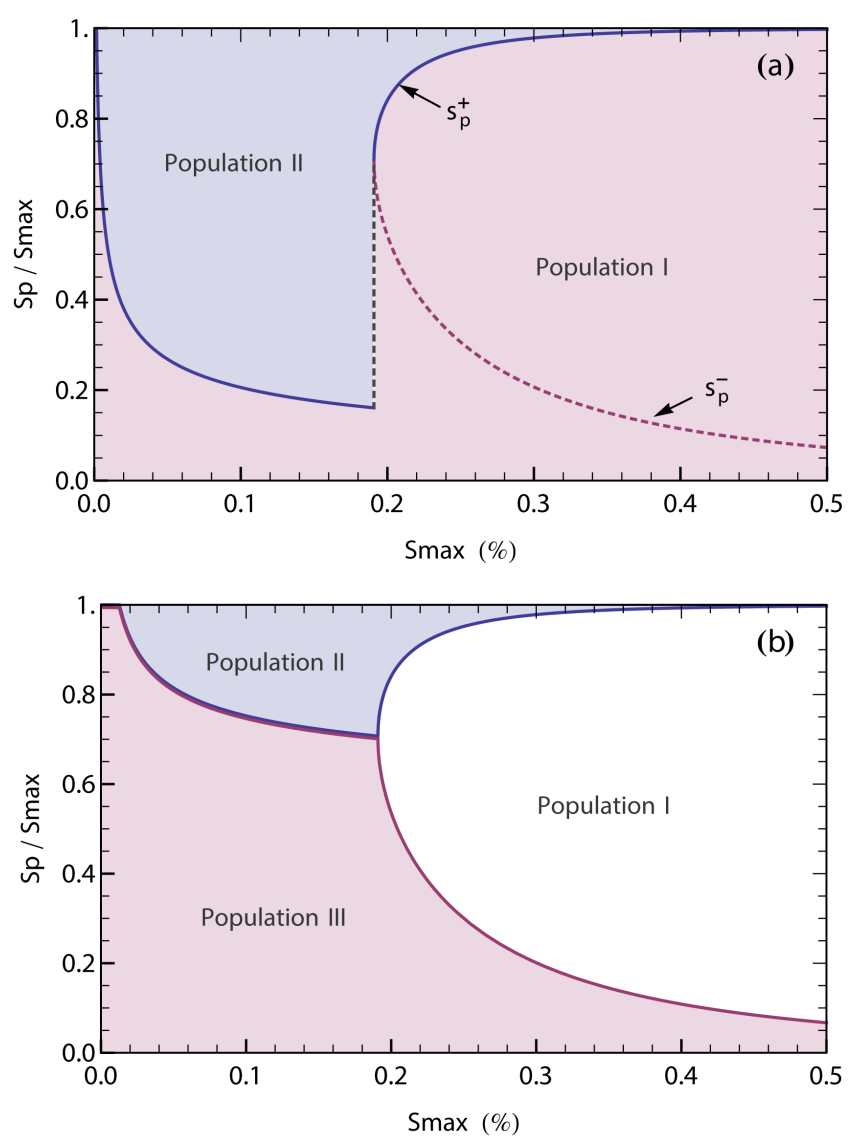

Figure 1. The "partitioning supersaturations" $s_{\mathrm{p}}^{ \pm}$illustrated in the $s_{\mathrm{C}}-s_{\text {max }}$ space. (a) The $s_{\mathrm{c}}-s_{\max }$ space as used in Nenes and Seinfeld (2003) and Barahona et al. (2010); and (b) as used in this study. The example here is for a vertical velocity $w=0.1 \mathrm{~ms}^{-1}$.

II"), $D_{\mathrm{p}}$ was approximated by $D_{\mathrm{pc}}$. This is a reasonable assumption, since these small particles are the most likely to equilibrate instantaneously with the ambient supersaturation and, as discussed before, they have little time to grow. For those CCN with $s_{\mathrm{p}}^{+}>s_{\mathrm{c}}>s_{\mathrm{p}}^{-}$(termed here as "population I"), approximation $D_{\mathrm{p}}^{(2)}$ was used. This still leaves a third population out: the large $\mathrm{CCN}$ with $s_{\mathrm{c}}<s_{\mathrm{p}}^{-}$. Despite the rules of Eq. (10) dictating that, for this population, $D_{\mathrm{p}}^{(1)}>D_{\mathrm{p}}^{(2)}$, it is well known that, in actuality, they are generally not capable of growing at equilibrium, so their size at $s_{\max }$ is much smaller than their $D_{\mathrm{pc}}$. Using these arguments, the large CCN particles were merged together into population I by using approximation $D_{\mathrm{p}}^{(2)}$ for all particles with $s_{\mathrm{c}}<s_{\mathrm{p}}^{+}$, i.e., discarding $s_{\mathrm{p}}^{-}$(Fig. 1a).

The approach was completed by defining an empirically derived $s_{\mathrm{p}}$ for the regime where $s_{\max }<\xi_{\mathrm{c}}$ (and Eq. 10 admits only imaginary solutions). This is:

$\frac{s_{\mathrm{p}}^{+}}{s_{\max }}=\frac{2 \times 10^{7}}{3} A s_{\max }^{-0.3824}$. 

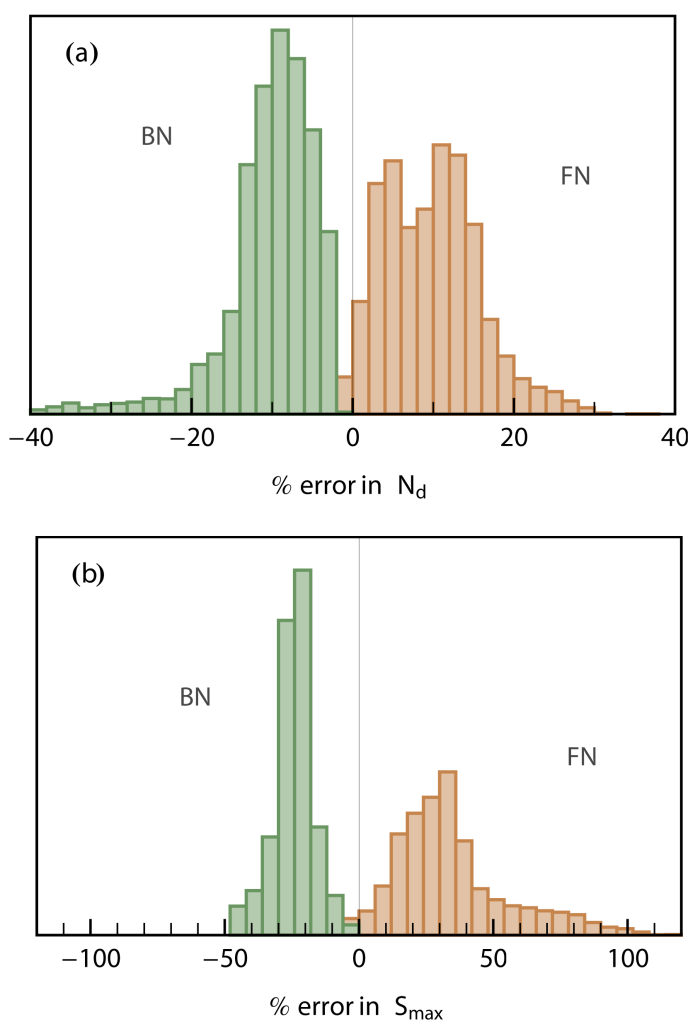

Figure 2. Histogram of the frequency of occurrence for the relative error $\epsilon=1-\chi_{\text {param }} / \chi_{\mathrm{PM}}$, where $\chi_{\text {param }}$ is the parameterized value, and $\chi_{\mathrm{PM}}$ is the value from parcel-model simulations. (a) for the droplet number $\left(N_{\mathrm{d}}\right)$, (b) for the maximum supersaturation $\left(s_{\max }\right)$.

The population-splitting formulation has been shown to have great skill in capturing the behavior of $s_{\max }$ under a large set of aerosol and updraft inputs. The Fountoukis and Nenes (2005) parameterization (FN hereafter), which is based on the framework described above, has also been capable of reproducing observed cloud-droplet concentrations (e.g., Meskhidze et al., 2005; Fountoukis et al., 2007).

\subsubsection{Correction for inertially limited CCN}

Based on detailed numerical simulations of the activation process, Barahona et al. (2010) noted that, when the activation process occured in situations of weak updrafts and the aerosol contains a significant number of large $\mathrm{CCN}$, the FN parameterization exhibited a tendency to overestimate $s_{\max }$ and $N_{\mathrm{d}}$. It was shown that this behavior originated in the assumptions made regarding the size of the inertially limited $\mathrm{CCN}$. By analyzing the first-order derivatives of the FN parameterization with respect to input parameters, Morales Betancourt and Nenes (2014) further confirmed a lack of sensitivity of $N_{\mathrm{d}}$ computed with FN to perturbations in the properties of coarse-mode particles, i.e., to number concentration, mode diameter and hygroscopicity parameter. This indicated that, although the total droplet number was not drastically affected by the coarse-mode-aerosol properties, the slight overestimation of $N_{\mathrm{d}}$ and $s_{\max }$, mentioned above, was due to the underestimation of the water-vapor depletion by the large $\mathrm{CCN}$ population.

A simple correction term for these inertially limited droplets was introduced by Barahona et al. (2010). As the timescale for large soluble particles (whose equilibrium supersaturation follows the Köhler Eq. A4) to grow to $D_{\mathrm{pc}}$ is many times larger than the timescale of cloud formation, and therefore this size is not reached by the inertially limited $\mathrm{CCN}$, it was proposed that the condensation rate on this population could be estimated by approximating their size at $s_{\max }$ with their equilibrium diameter at $s=0, D_{\mathrm{p} 0}$. Using Köhler theory, it can be shown that the equilibrium wet diameter of a particle when exposed to $100 \%$ relative humidity is equal to $D_{\mathrm{pc}} / \sqrt{3}$ (Barahona et al., 2010). This third approach to the diameter of the growing droplets is denoted here by $D_{\mathrm{p}}^{(3)}$. The correction term proposed by Barahona et al. (2010) consisted, then, of adding $D_{\mathrm{p}}^{(2)}$ and $D_{\mathrm{p}}^{(3)}$ concurrently to estimate the size at $s_{\max }$. This approximation was applied to all the particles with $s_{\mathrm{c}}<s_{\mathrm{p}}^{+}$, i.e., to all the population I particles depicted in Fig. 1a.

In this work, we show that the approach of Barahona et al. (2010) inadvertently overestimates the size for the population I particles. Equation (6) for $D_{\mathrm{p}}$ involves the square root of the sum of the growth term and the initial size, therefore directly adding both term results in an overestimation bias for $D_{\mathrm{p}}$, and a corresponding overestimation of the contribution of this population to the condensation rate. Therefore, it is necessary to revise the population-splitting concept to consistently combine the contributions from all $\mathrm{CCN}$ to the condensation rate.

Up until now, our discussion has relied on the assumption that particles activate in accordance with Köhler's theory. However, insoluble particles, such as uncoated mineral dust and volcanic ash, for which activation follows the adsorption activation theory (Sorjamaa and Laaksonen, 2007; Kumar et al., 2009; Lathem et al., 2011), tend to uptake considerably less water before activation than Köhler particles. As shown by Kumar et al. (2009), the ratio between the critical wet diameter $D_{\mathrm{pc}}$ and the dry aerosol diameter $d_{\mathrm{p}}$ for insoluble particles is less than 2 for most conditions, and this ratio is only weakly dependent on the size of the dry particle (see Appendix A). For this reason, insoluble particles that activate via adsorption activation are typically capable of growing at equilibrium with the ambient supersaturation, reaching their $D_{\mathrm{pc}}$, and the mechanisms of kinetic limitations are different to those outlined in Barahona et al. (2010). Furthermore, the behavior of insoluble particles, as explored by Kumar et al. (2009), considers that, independently of their size, all insoluble particles are capable of reaching $D_{\mathrm{pc}}$, and the contribution from growth dominates at all particles sizes, which implies that the population-splitting concept is not necessary for these particles. For these reasons, the revision 
of the population-splitting concept is limited to particles activating in accordance with Köhler's theory.

\subsection{The population-splitting concept revisited}

We aim to improve two main aspects of the parameterization framework of Nenes and Seinfeld (2003) and Barahona et al. (2010). First, we aim to better account for the size of inertially limited CCN, so that their contribution to supersaturation depletion can be quantified correctly. The second goal is to avoid the discontinuity in $s_{\mathrm{p}}^{ \pm}$introduced in Eq. (11). As $s_{\max }$ approaches $\xi_{\mathrm{c}}$ from above, $s_{\mathrm{p}}^{ \pm}$from Eq. (10) approaches $1 / \sqrt{2}$. However, the value of $s_{\mathrm{p}}^{+}$for $s_{\max }=\xi_{\mathrm{c}}$ in Eq. (11) is in general, not equal to $1 / \sqrt{2}$. This implies a discontinuity in the calculation of the surface area of droplets, which, in turn, creates a discontinuity in the parameterization response in scenarios where $s_{\max }$ shifts from the $s_{\max }<\xi_{\mathrm{c}}$ regime to the $s_{\max }>\xi_{\mathrm{c}}$.

The first goal is attained by recognizing, as Barahona et al. (2010) did, that neither $D_{\mathrm{p}}^{(1)}$ or $D_{\mathrm{p}}^{(2)}$ are appropriate approximations for the size of the largest $\mathrm{CCN}$ particles. However, instead of merging all CCN with $s_{\mathrm{c}}<s_{\mathrm{p}}^{+}$in the same population (population I in Fig. 1a), we consider that only the largest particles - those with $s_{\mathrm{c}}<s_{\mathrm{p}}^{-}$- should be approximated as in Barahona et al. (2010), i.e., $D_{\mathrm{p}}^{(3)} \approx D_{\mathrm{pc}} / \sqrt{3}$ (Fig. 1b). Similarly, and to maintain consistency and avoid overestimation of the water uptake, $D_{\mathrm{p}}$ for CCN with $s_{\mathrm{p}}^{+}>s_{\mathrm{c}}>s_{\mathrm{p}}^{-}$are approximated with Eq. (9):

$$
\begin{array}{ll}
D_{\mathrm{p}}^{(1)} \approx D_{\mathrm{pc}}\left(s_{\mathrm{c}}\right)=\frac{2 A}{3 s_{\mathrm{c}}} & s_{\mathrm{c}}>s_{\mathrm{p}}^{+} \\
D_{\mathrm{p}}^{(2)} \approx\left(\frac{G}{\alpha w}\right)^{1 / 2}\left(s_{\max }^{2}-s_{\mathrm{c}}^{2}\right)^{1 / 2} & s_{\mathrm{p}}^{+}>s_{\mathrm{c}}>s_{\mathrm{p}}^{-} \\
D_{\mathrm{p}}^{(3)} \approx D_{\mathrm{p} 0}\left(s_{\mathrm{c}}\right)=\frac{2 A}{3 \sqrt{3} s_{\mathrm{c}}} & s_{\mathrm{c}}<s_{\mathrm{p}}^{-},
\end{array}
$$

and the integral $I\left(0, s_{\max }\right)$ is naturally split in the different contributions:

$s_{\max }\left[I\left(0, s_{\mathrm{p}}^{-}\right)+I\left(s_{\mathrm{p}}^{-}, s_{\mathrm{p}}^{+}\right)+I\left(s_{\mathrm{p}}^{+}, s_{\max }\right)\right]=\beta$.

The computation of Eq. (13) can be done either discretely, by splitting the CCN spectra in sections or bins (e.g., Nenes and Seinfeld, 2003; Fountoukis and Nenes, 2005), or continously, if the $\mathrm{CCN}$ spectra can be written as a lognormal distribution (e.g., Fountoukis and Nenes, 2005; Barahona et al., 2010).

The second goal is achieved by defining the partition supersaturation for $s_{\max }<\xi_{\mathrm{c}}$, such that it transitions smoothly to the regime, where $\mathrm{CCN}$ is completely dominated by inertially limited particles. Noting that, as $s_{\max } \rightarrow \xi_{\mathrm{c}}$, both roots become identical $s_{\mathrm{p}}^{+}=s_{\mathrm{p}}^{-}$, and both approach the value

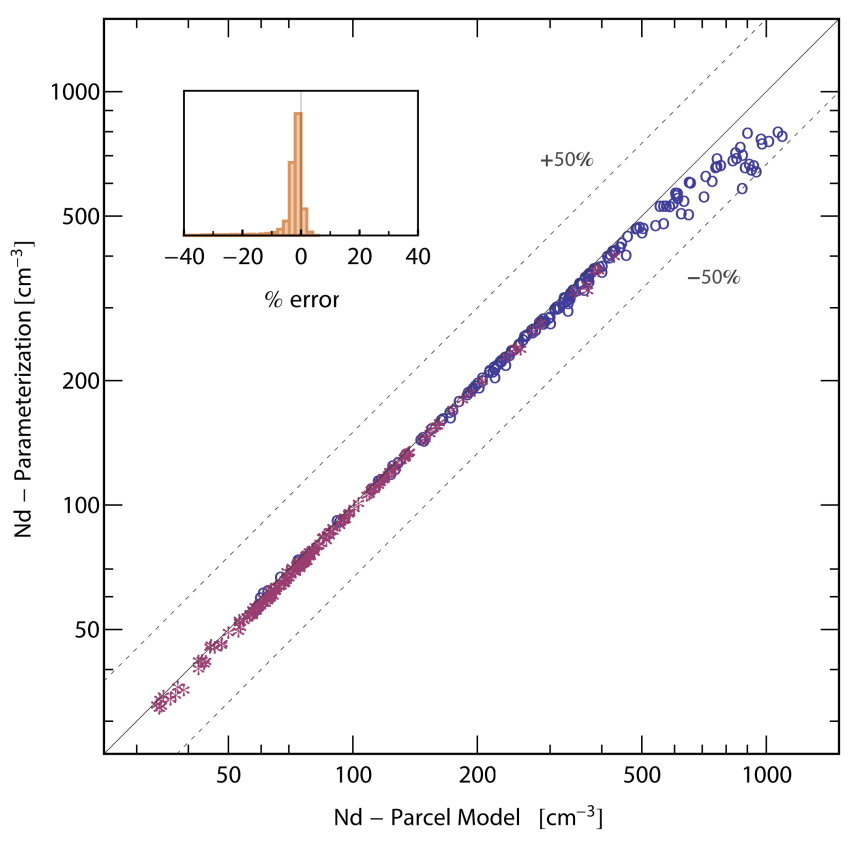

Figure 3. Comparison between parcel-model simulations and parameterization results. Blue circles correspond to continental aerosol, while red stars are for marine aerosol. The inset is a histogram of the relative error between parcel model and parameterization-derived $N_{\mathrm{d}}$.

$1 / \sqrt{2}$, we define $s_{\mathrm{p}}$ as

$\frac{s_{\mathrm{p}}^{ \pm}}{s_{\max }}=\frac{2 A \times 10^{7}}{3}\left(s_{\max }^{-0.3824}-\xi_{\mathrm{c}}^{-0.3824}\right)+\frac{1}{\sqrt{2}}$,

which maintains the same empirically derived dependence on $s_{\max }$, but solves the discontinuity issue in the original framework of Nenes and Seinfeld (2003). From this expression, the vanishing of the term $I\left(s_{\mathrm{p}}^{-}, s_{\mathrm{p}}^{+}\right)$emerges naturally for $s_{\max }<\xi_{\mathrm{c}}$, since both roots collapse to the same value. The regions in which each approximation should be used are depicted in Fig. 1b.

\subsection{Numerical implementation}

The modifications proposed here can be implemented in the existing Barahona et al. (2010) framework without the need of any major changes. Using the functions $I_{1}\left(0, s_{\mathrm{p}}\right)$ and $I_{2}\left(s_{\mathrm{p}}, s_{\max }\right)$, whose formulas are given in Nenes and Seinfeld (2003) for sectional, and in Fountoukis and Nenes (2005) for lognormal aerosol-size distribution (see Appendix B), $I\left(0, s_{\max }\right)$ is simply given by the following expression:

$$
\begin{aligned}
I\left(0, s_{\max }\right)= & \frac{1}{\sqrt{3}} I_{2}\left(0, s_{\mathrm{p}}^{-}\right) \\
& +\left[I_{1}\left(0, s_{\mathrm{p}}^{+}\right)-I_{1}\left(0, s_{\mathrm{p}}^{-}\right)\right]+I_{2}\left(s_{\mathrm{p}}^{+}, s_{\max }\right),
\end{aligned}
$$

which can be implemented with minimal adjustments to codes that use the original population-splitting concept. This 
expression can be extended to the formulation of Barahona and Nenes (2007) that includes the effects of entrainment and mixing in the supersaturation development. If subsaturated air entrains the air parcel at a fractional entrainment rate $\mu$, the condensation rate onto the droplets is reduced, and Eq. (5) transforms to

$s_{\max } I\left(0, s_{\max }\right)=\beta\left(1-\mu / \mu_{\mathrm{c}}\right)$,

where $\mu_{\mathrm{c}}$ is the "critical entrainment rate", defined in Barahona and Nenes (2007) as the entrainment rate that prevents the cloud parcel from generating water-vapor supersaturation, and is given by

$\mu_{\mathrm{c}}=\frac{\alpha}{1-\mathrm{RH}}\left(1-\frac{L_{\mathrm{v}} M_{\mathrm{w}} \Delta T}{R T^{2}}\right)^{-1}$,

where RH is the relative humidity of the entrained air, and $\Delta T=T-T^{\prime}$ is the difference between the parcel and entrained-air temperatures.

\section{Results}

In this section, we present the results of an evaluation of the parameterization performance against predictions of $s_{\max }$ and $N_{\mathrm{d}}$ computed with a detailed numerical parcel model of the condensation growth of droplets. Three different versions of the parameterization framework of Nenes and Seinfeld (2003) are evaluated here: the Fountoukis and Nenes (2005)(FN), the Barahona et al. (2010)(BN) and, finally, the new parameterization proposed in this paper.

\subsection{Aerosol and updraft velocity fields}

The augmented parameterization presented in this work was tested against computations of $N_{\mathrm{d}}$ and $s_{\max }$ from a detailed numerical parcel model of the condensational growth of droplets (Nenes et al., 2001). In order to explore the parameterization in the conditions typically encountered in a GCM simulation, we employed offline annual average aerosol fields and cloud-scale vertical velocity from a climatological simulation performed by Morales Betancourt and Nenes (2014) with the Community Atmospheric Model 5.1 (CAM5.1). The simulations correspond to present-day aerosol emissions from the Lamarque et al. (2010) emission inventory. CAM5.1 includes a 3-mode lognormal aerosol model, MAM3 (Liu et al., 2012). The aerosol fields used in this study correspond to the $930 \mathrm{hPa}$ pressure level, and include only the grid cells between $75^{\circ} \mathrm{N}$ and $75^{\circ} \mathrm{S}$, totaling 9504 instances of aerosol-size distributions, chemical composition, and updraft velocity, each corresponding to one of the model grid cells considered. The fields used to drive the parameterization and parcel-model simulations include the aerosol-number concentration for each lognormal mode, $n_{\mathrm{a}_{i}}$, the hygroscopicity parameter, $\kappa_{\mathrm{a}_{i}}$, and the geometric mean diameter $d_{\mathrm{g}_{i}}$, for each mode. The hygroscopicity parameter
Table 1. Size distribution parameters for the 3-modal aerosol-size distribution used for evaluation of the parameterization. Each lognormal mode is characterized by the number concentration $n_{\mathrm{a}_{i}}$, geometric standard deviation $\sigma_{\mathrm{g}_{i}}$, geometric mean diameter $d_{\mathrm{g}_{i}}$, and the hygroscopicity of the mode $\kappa_{\mathrm{a}_{i}}$.

\begin{tabular}{lcccc}
\hline Aerosol mode & $\sigma_{\mathrm{g}_{i}}$ & $\begin{array}{c}n_{\mathrm{a}_{i}} \\
\left(\mathrm{~cm}^{-3}\right)\end{array}$ & $\begin{array}{c}d_{\mathrm{g}_{i}} \\
(\mu \mathrm{m})\end{array}$ & $\kappa_{\mathrm{a}_{i}}$ \\
\hline (1) - Aitken & 1.6 & $40-200$ & $0.004-0.055$ & $0.37-0.72$ \\
$(2)-$ Accumulation & 1.8 & $30-510$ & $0.13-0.35$ & $0.18-1.05$ \\
$(3)$ - Coarse & 1.8 & $0.1-5.0$ & $1.0-4.0$ & $0.11-1.16$ \\
\hline
\end{tabular}

is computed in CAM5.1 from the chemical composition of the aerosol. Accumulation-mode aerosol includes six aerosol species (sulfate, primary and secondary organic matter, black carbon, sea salt and dust). The Aitken mode contains sulfate, secondary organic aerosol and sea salt. The coarse mode includes sulfate, sea salt and dust. The grid-cell average cloudscale vertical velocity, $w$, was used as input for the simulations. The CAM aerosol fields are described in detail by Liu et al. (2012). The ranges over which the parameters of the test aerosol fields are explored in this work are reported in Table 1.

\subsection{Cloud-parcel-model configuration}

A numerical solution to the equations describing the condensational growth of a population of droplets was performed with a numerical cloud parcel model. The details of the model can be found elsewhere (e.g., Nenes et al., 2001), but here we include a description of the configuration used in this work. The modeling framework is that of an adiabatic Lagrangian air parcel moving vertically with a constant updraft velocity, $w$. The state of the air parcel is described by its temperature $T$, pressure $p$, and the mixing ratios of liquid water and water vapor, $q_{1}$ and $q_{\mathrm{v}}$, respectively. The droplet population is separated into size bins, with the center diameter of each bin allowed to grow or shrink as the condensation or evaporation process proceeds. The water mixing ratio $q_{1}$ is expressed as

$q_{1}(t)=\frac{\pi}{6} \frac{\rho_{\mathrm{l}}}{\rho_{\mathrm{a}}} \sum_{i} N_{i} D_{\mathrm{p}_{i}}^{3}$,

where $N_{i}$ is the number of droplets in the size category $i$, and $D_{\mathrm{p}_{i}}$ is the size of the droplets in size category $i$ at time $t$. The mass transfer from the vapor to the droplets is explicitly calculated using the droplet growth equation. In this application, 35 logarithmically spaced size bins were employed for each lognormal mode, totaling 105 size bins. The binning method ensures that $99.5 \%$ of the particles in each lognormal mode are accounted for in the simulation. As initial condition, it was assumed that the wet aerosol particles were at equilibrium with a $90 \%$ ambient relative humidity. In order to be consistent with the approach taken in the 
Table 2. Summary of comparisons against parcel-model simulations expressed as $\epsilon \pm \sigma_{\epsilon}$.

\begin{tabular}{lcccc}
\hline $\begin{array}{l}\text { Activation } \\
\text { Parameterization }\end{array}$ & $s_{\max }$ & $N_{\mathrm{d}}$ & $\mathrm{d} N_{\mathrm{d}} / \mathrm{d} n_{\mathrm{a}}$ \\
\hline Fountoukis and Nenes (2005) & $\mathrm{FN}$ & $+31 \% \pm 25 \%$ & $+7.8 \% \pm 9.7 \%$ & $+8.7 \% \pm 30.2 \%$ \\
Barahona et al. (2010) & $\mathrm{BN}$ & $-24 \% \pm 7 \%$ & $-10 \% \pm 7.8 \%$ & $-19.5 \% \pm 17.6 \%$ \\
This work & & $-6.0 \% \pm 6.2 \%$ & $-2.7 \% \pm 4.8 \%$ & $-9.3 \% \pm 12.1 \%$ \\
\hline
\end{tabular}

parameterization, a droplet is considered to be activated if their critical supersaturation $s_{\mathrm{c}}$ is lower than the maximum supersaturation $s_{\max }$. Overall, the parcel model solves equations for the droplet size for each size bin, $D_{\mathrm{p}_{i}}$, temperature $T$, pressure $p$ and supersaturation $s$. The updraft velocity $w$ was assumed constant in these integrations.

\subsection{Parameterization evaluation}

As shown by Morales Betancourt and Nenes (2014), the firstorder derivatives of the parameterized $N_{\mathrm{d}}$, with respect to input parameters, are useful in understanding the parameterization ability to respond to perturbations to the input variables. Therefore, in addition to evaluating the parameterized $N_{\mathrm{d}}$ and $s_{\text {max }}$ against those of parcel-model simulations, we also performed calculations of the first-order derivatives of the parameterized $N_{\mathrm{d}}$ with respect to any input variable $\chi_{j}$, $\partial N_{\mathrm{d}} / \partial \chi_{j}$. These sensitivity calculations were then compared against finite difference approximation to the derivatives with the numerical cloud parcel model. We report the mean of the relative error $\epsilon$ and the standard deviation of the errors $\sigma_{\epsilon}$ between parameterization predictions of $N_{\mathrm{d}}$ and $s_{\max }$, and those computed with the parcel model. We performed this analysis for each of the 9504 cases considered.

The results show a significant improvement in the accuracy and precision of the parameterized $N_{\mathrm{d}}$ and $s_{\max }$ values, without any appreciable increase in the computational cost. Table 2 summarize the results of the performance evaluation for the various parameterizations considered here. Figure $2 \mathrm{a}$ and $2 \mathrm{~b}$ show the distribution of errors for $s_{\max }$ and $N_{\mathrm{d}}$ for $\mathrm{FN}$ and $\mathrm{BN}$. When $\mathrm{FN}$ was used, the relative error in $s_{\max }$ was, on average, $+31 \% \pm 25 \%$, while, that in $N_{\mathrm{d}}$ was of $+7.8 \% \pm 9.7 \%$. Both numbers indicate a moderate overestimation in both fields for the conditions explored. The same analysis for the Barahona et al. (2010) parameterization shows a relative error for $s_{\max }$ of $-24 \% \pm 7 \%$, and $N_{\mathrm{d}}$ of $-10 \% \pm 7.8 \%$, showing a small underestimation of both fields under the conditions explored in the simulations (2). For both fields, there is a marked decrease in both the average error (a measure of parameterization bias) and in the dispersion of the errors (a measure of the parameterization accuracy). Figure 3 shows the results of the comparison between $N_{\mathrm{d}}$ computed with the parameterization developed in this work and the parcel model. The relative error, when applying the modifications proposed in this work, was considerably lower, being $-6.0 \% \pm 6.2 \%$ for $s_{\max }$, and $-2.7 \% \pm 4.8 \%$ for $N_{\mathrm{d}}$. The errors of the sensitivity to total aerosol perturbation, $\mathrm{d} N_{\mathrm{d}} / \mathrm{d} n_{\mathrm{a}}$, computed with the parameterization presented here and with the parcel model exhibited a decrease in the bias, such as that shown by $N_{\mathrm{d}}$ and $s_{\max }$ (Table 2).

A summary of the mean relative errors of the sensitivities $\partial N_{\mathrm{d}} / \partial \chi_{j}$ for the Barahona et al. (2010) and for the parameterization presented in this work are shown in Fig. 4. The modifications introduced here result in a higher sensitivity to aerosol-number concentration when compared to $\mathrm{BN}$ for the three modes considered. Figure $4 \mathrm{a}$ suggests that most of the improvement in the ability to predict $s_{\max }, N_{\mathrm{d}}$ and $\mathrm{d} N_{\mathrm{d}} / \mathrm{d} n_{\mathrm{a}}$ is due to a better representation of the response to accumulation-mode particles. As such, the mean error for $\partial N_{\mathrm{d}} / n_{\mathrm{a}_{i}}$ of the accumulation mode went from an average of $-9.4 \%$ for BN to only $-0.6 \%$. Since this mode represents the bulk of the $\mathrm{CCN}$ population, any changes to the representation of its water uptake have great impact on $s_{\max }$ and $N_{\mathrm{d}}$. Figure 4 also shows that the magnitude of the mean errors for the Aitken and the accumulation-mode sensitivity to $n_{\mathrm{a}}$, $\kappa_{\mathrm{a}}$ and $d_{\mathrm{g}}$ are smaller for the parameterization presented here. Nevertheless, it can also be seen that the modifications introduced here result in an overestimation of the sensitivities of these variables for the coarse-mode particles. The sensitivity of $N_{\mathrm{d}}$ to $\kappa_{\mathrm{a}_{i}}$ and $d_{\mathrm{g}_{i}}$ show the ability of the parameterization to respond to changes in the chemical composition of the aerosol and to the total aerosol volume. Both quantities, the hygroscopicity parameter and the aerosol size, directly impact the critical supersaturation. Therefore, changes in these parameters have an impact on the water-vapor sink, and control the maximum supersaturation attained in the parcel.

\section{Summary and conclusions}

The population-splitting concept of Nenes and Seinfeld (2003) and Barahona et al. (2010) was further developed to consistently account for the condensation rate of inertially limited $\mathrm{CCN}$. The modifications to this parameterization framework were shown to improve the accuracy and precision for predictions of maximum supersaturation $s_{\max }$, and cloud-droplet-number concentration $N_{\mathrm{d}}$. Similarly, the sensitivity of the parameterized $N_{\mathrm{d}}$ to aerosol-number concentration, $\mathrm{d} N_{\mathrm{d}} / \mathrm{d} n_{\mathrm{a}}$, was found to be in better agreement when compared to detailed numerical simulations of the activation process. The first-order derivatives $\partial N_{\mathrm{d}} / \partial \chi_{j}$ of the parameterized $N_{\mathrm{d}}$ were also compared against numerical 

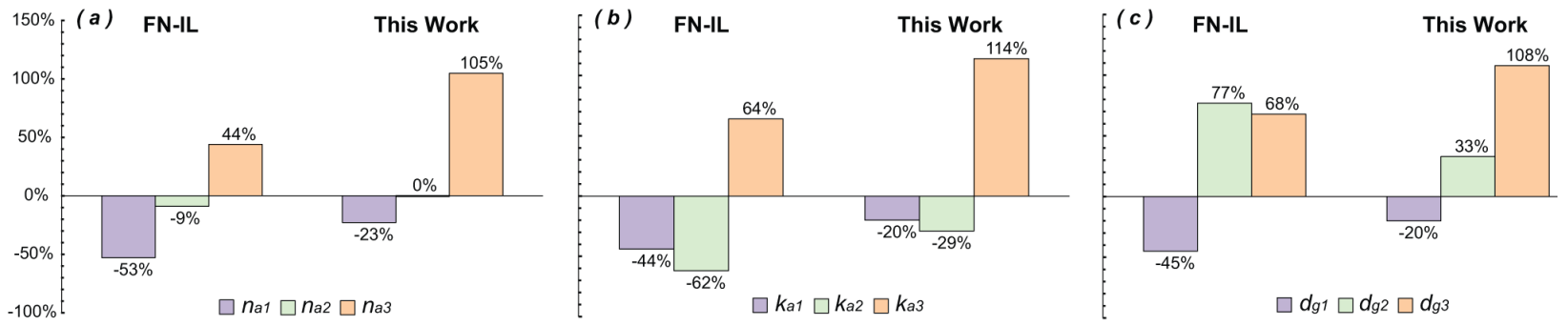

Figure 4. Mean relative percent error, $\epsilon$, between sensitivities computed with the detailed parcel-model simulations and the parameterization results. Comparisons are shown here for (a) sensitivity to aerosol number $\partial N_{\mathrm{d}} / \partial n_{\mathrm{a}_{i}}$, (b) sensitivity to the hygroscopicity parameter $\partial N_{\mathrm{d}} / \partial \kappa_{\mathrm{a}_{i}}$, (c) sensitivity to aerosol geometric mean diameter $\partial N_{\mathrm{d}} / \partial d_{\mathrm{g}_{i}}$. Comparisons are shown for the BN parameterization and the results of this work. Sub-indices follow the notation of Table 1.

parcel-model estimates. This analysis showed that the modifications presented here result in a more consistent response to perturbations to the characteristics of Aitken and accumulation-mode particles, while revealing a slight overrepresentation of the response to coarse-mode-aerosol properties. Implementation of these modifications to the population-splitting framework is straightforward and does not require any major modifications to the previous formulations. This minor code change comes at no additional computational expense, and produces virtually identical results to a numerical parcel model, both in terms of $N_{\mathrm{d}}$ and sensitivities $\partial N_{\mathrm{d}} / \partial \chi_{j}$. The impact of these changes is expected to be larger in environments dominated by highly hygroscopic coarse-mode aerosol, such as marine environments far from pollution sources, as well as regions with a large number of accumulation-mode particles. 


\section{Appendix A: Notation}

The functions $\alpha$ and $\gamma$ from Eq. (1) are given by

$\alpha=\frac{g L_{\mathrm{v}} M_{\mathrm{w}}}{c_{\mathrm{p}} R T^{2}}-\frac{g M_{\mathrm{a}}}{R T}$

and

$\gamma=\frac{L_{\mathrm{v}}^{2} M_{\mathrm{w}}}{c_{\mathrm{p}} R T^{2}}+\frac{M_{\mathrm{a}} p}{M_{\mathrm{w}} e_{\mathrm{s}}}$,

where $T$ is the temperature of the air parcel, $e_{\mathrm{S}}$ is the saturation vapor pressure, $g$ is the gravitational constant, $L_{\mathrm{v}}$ is the latent heat of vaporization of water, $c_{\mathrm{p}}$ is the heat capacity of air, $R$, the universal gas constant and $M_{\mathrm{a}}$ and $M_{\mathrm{w}}$ are the molecular weights of air and water respectively.

The function $G$ in the droplet growth equation is given by

$G=4\left[\frac{\rho_{\mathrm{w}} R T}{e_{\mathrm{s}} D_{\mathrm{v}} M_{\mathrm{w}}}+\frac{L_{\mathrm{v}} \rho_{\mathrm{w}}}{k_{\mathrm{a}} T}\left(\frac{L_{\mathrm{v}} M_{\mathrm{w}}}{R T}-1\right)\right]^{-1}$,

where $\rho_{\mathrm{a}}$ and $\rho_{\mathrm{w}}$ are the density of air and water respectively, $D_{\mathrm{v}}$ is the water-vapor diffusivity and $k_{\mathrm{a}}$ is the thermal conductivity of air. The equation describing the equilibrium supersaturation over the surface of a water droplet containing a solute is given by the Köhler equation:

$s_{\mathrm{eq}}=\frac{A}{D_{\mathrm{p}}}-\kappa \frac{d_{\mathrm{p}}^{3}}{D_{\mathrm{p}}^{3}}$,

where $\kappa$ is the hygroscopicity parameter (Petters and Kreidenweis, 2007), and the coefficient $A$ is related to the dropletsurface tension $\sigma$ as $A=4 M_{\mathrm{w}} \sigma / R T$. The critical supersaturation $s_{\mathrm{c}}$, i.e., the maximum of Eq. (A4), also defines the critical diameter, $D_{\mathrm{pc}}, s_{\mathrm{eq}}\left(D_{\mathrm{pc}}\right)=s_{\mathrm{c}}$. By setting $\mathrm{d} s_{\mathrm{eq}} / \mathrm{d} D_{\mathrm{p}}=0$ and solving for $D_{\mathrm{p}}$, it can be seen that the critical diameter is related to $s_{\mathrm{c}}$ as $D_{\mathrm{pc}}=2 A / 3 s_{\mathrm{c}}$. Similarly, the dry diameter $d_{\mathrm{p}}$ can be related to its corresponding critical supersaturation $s_{\mathrm{c}}$ (e.g., Seinfeld and Pandis, 2006):

$s_{\mathrm{c}}=\left(\frac{4 A^{3}}{27 \kappa}\right)^{1 / 2} d_{\mathrm{p}}^{-3 / 2}$.

The power-law relationship between $s_{\mathrm{c}}$ and $d_{\mathrm{p}}$ of Eq. (A5) implies that $D_{\mathrm{pc}}$ grows as $d_{\mathrm{p}}^{3 / 2}$ for soluble particles (following Köhler's theory), and the ratio $D_{\mathrm{pc}} / d_{\mathrm{p}}$ increases with aerosol size as $\sim d_{\mathrm{p}}^{1 / 2}$. For insoluble particles such as dust, a few layers of water molecules are adsorbed onto the aerosol surface at subsaturated conditions, resulting in equilibrium wet diameters that are similar to the dry aerosol diameter. Kumar et al. (2009) derived a relation equivalent to Eq. (A5) for insoluble particles, and expressed it as $s_{\mathrm{c}} \approx c d_{\mathrm{p}}^{-x}$, with $c$ and $x$ being empirically derived quantities. The exponent $x$ ranges between 0.8 and 1 . This value for the exponent $x$ for insoluble particles implies that the ratio $D_{\mathrm{pc}} / d_{\mathrm{p}}$ decreases slightly with increasing $d_{\mathrm{p}}$.

\section{Appendix B: Summary of changes in existing codes}

The conceptual approach for all of the parameterization discussed here involves the same steps and require an iterative solution of Eq. (5). Fundamental to the computation of $N_{\mathrm{d}}$ is to determine the number of particles that would activate as a function of supersaturation, $N_{\mathrm{CCN}}$, and is represented by a cumulative $\mathrm{CCN}$ spectrum $F(s)$. In the case where the aerosol-size distribution is described by $n_{\mathrm{m}}$ lognormal modes, $F(s)$ is given by

$F(s)=\sum_{i}^{n_{\mathrm{m}}} \frac{n_{\mathrm{ai}}}{2} \operatorname{erfc}\left(u_{i}(s)\right)$,

where $\operatorname{erfc}(x)=1-\operatorname{erf}(x)$ is the complement error function, $n_{\mathrm{m}}$ is the number of modes in the aerosol-size distribution and $n_{\mathrm{a}_{i}}$ is the number concentration corresponding to mode $i$. The function $u_{i}$ is given by

$u_{i}(s)=\frac{2 \ln \left(s_{\mathrm{g}_{i}} / s\right)}{3 \sqrt{2} \ln \sigma_{\mathrm{g}_{i}}}$,

where $s_{\mathrm{g}_{i}}$ is the critical supersaturation corresponding to the geometric mean diameter $d_{\mathrm{g}_{i}}$ of the mode.

The conceptual steps in the solution are as follows:

1. Guess an initial value for $s_{\max }$.

2. Compute $\xi_{\mathrm{c}}=\left(16 A^{2} \alpha w / 9 G\right)^{1 / 4}$ (or equivalently $\Delta=$ $\left.1-\xi_{\mathrm{c}}^{4} / s_{\max }^{4}\right)$.

3. Evaluate $\xi_{\mathrm{c}}$ (or $\Delta$ ) to determine the corresponding partitioning supersaturations $s_{\mathrm{p}}^{ \pm}$.

4. Compute the integral $I\left(0, s_{\max }\right)$.

5. Evaluate the expression: $s_{\max } I\left(0, s_{\max }\right) \stackrel{?}{=} \beta$ (Eq. 5).

6. If convergence is met in step $5, N_{\mathrm{d}}=F\left(s_{\max }\right)$. If convergence is not met, repeat steps 1 to 5 .

Existing parameterization codes involve minimal changes in steps 3 and 4 alone. We specifically address the necessary changes to codes that follow the Fountoukis and Nenes (2005) and the Barahona et al. (2010). Alternatively, codes are available upon request to the authors.

This formulation of Fountoukis and Nenes (2005) involves the computation of only one partitioning supersaturation, $s_{\mathrm{p}}^{+}$, corresponding to the larger of the two roots in Eq. (10). If $\xi_{\mathrm{c}}>s_{\max }$, then $s_{\mathrm{p}}^{+}$is computed from the positive root in Eq. (10). If $\xi_{\mathrm{c}}<s_{\max }$, then $s_{\mathrm{p}}^{+}$computed using Eq. (11). The integral $I\left(0, s_{\max }\right)$ takes the form:

$I\left(0, s_{\max }\right)=\left[I_{1}\left(0, s_{\mathrm{p}}^{+}\right)+I_{2}\left(s_{\mathrm{p}}^{+}, s_{\max }\right)\right]$, 
where, for lognormal aerosol,

$$
\begin{aligned}
& I_{1}\left(0, s_{\mathrm{p}}\right)=\frac{n_{\mathrm{a}_{i}}}{2}\left(\frac{G}{\alpha w}\right)^{1 / 2} \\
& s_{\max }\left[\operatorname{erfc}\left(u_{i}\left(s_{\mathrm{p}}\right)\right)-\frac{g_{i}}{2}\left(\frac{s_{\mathrm{g}_{i}}}{s_{\max }}\right)^{2} \operatorname{erfc}\left(u_{i}\left(s_{\mathrm{p}}\right)+\frac{3 \ln \sigma_{i}}{\sqrt{2}}\right)\right] \\
& I_{2}\left(s_{\mathrm{p}_{1}}, s_{\mathrm{p}_{2}}\right)=\frac{n_{\mathrm{a}_{i}}}{2} D_{\mathrm{g}_{i}} k_{i} \\
& {\left[\operatorname{erf}\left(u_{i}\left(s_{\mathrm{p}_{1}}\right)-\frac{3 \ln \sigma_{i}}{\sqrt{2}}\right)-\operatorname{erf}\left(u_{i}\left(s_{\mathrm{p}_{2}}\right)-\frac{3 \ln \sigma_{i}}{\sqrt{2}}\right)\right],}
\end{aligned}
$$

with $g_{i}=\exp \left(\frac{9}{2} \ln ^{2}\left(\sigma_{i}\right)\right), \quad k_{i}=\exp \left(\frac{9}{8} \ln ^{2}\left(\sigma_{i}\right)\right)$ and $D_{\mathrm{g}_{i}}=$ $2 A / 3 s_{\mathrm{g}_{i}}$ is defined as the critical wet diameter corresponding to the geometric mean diameter $d_{\mathrm{g}_{i}}$ for mode $i$.

Computation of the partitioning supersaturation following the correction for inertially limited CCN by Barahona et al. (2010) is identical as in the Fountoukis and Nenes (2005). The integral $I\left(0, s_{\max }\right)$, however, involves an extra term, and takes the form

$$
I\left(0, s_{\max }\right)=I_{1}\left(0, s_{\mathrm{p}}^{+}\right)+I_{2}\left(s_{\mathrm{p}}^{+}, s_{\max }\right)+\frac{1}{\sqrt{3}} I_{2}\left(0, s_{\mathrm{p}}^{+}\right),
$$

where the extra term $I_{2}\left(0, s_{\mathrm{p}}^{+}\right) / \sqrt{3}$ can be derived from Eq. (B5):

$$
I_{2}\left(0, s_{\mathrm{p}}\right)=\frac{n_{\mathrm{a}_{i}}}{2} D_{\mathrm{g}_{i}} k_{i}\left[\operatorname{erfc}\left(u_{i}\left(s_{\mathrm{p}}\right)-\frac{3 \ln \sigma_{i}}{\sqrt{2}}\right)\right] .
$$

The modifications introduced in this manuscript involve the computation of the partitioning supersaturations $s_{\mathrm{p}}^{ \pm}$. This computation is done in the following way:

$s_{\mathrm{p}}^{ \pm}=\left\{\begin{array}{l}\xi_{\mathrm{c}}>s_{\max } \longrightarrow s_{\mathrm{p}}^{ \pm} \text {from Eq. (10) } \\ \xi_{\mathrm{c}}<s_{\max } \longrightarrow s_{\mathrm{p}}^{+} \text {from Eq. (14). }\end{array}\right.$

Computation of the integral $I\left(0, s_{\max }\right)$ can be achieved by applying Eq. (15) using the expressions provided in Eqs. (B4), (B5) and (B7). 
Acknowledgements. We thank the DOE EaSM program for funding that supported the research carried out in this manuscript. Authors would like to thank Donifan Barahona for his valuable discussions on the manuscript.

Edited by: K. Gierens

\section{References}

Abdul-Razzak, H. and Ghan, S.: A parameterization of aerosol activation: 2. Multiple aerosol types, J. Geophys. Res., 105, 68376844, 2000.

Abdul-Razzak, H., Ghan, S., and Rivera-Carpio, C.: A parameterization of aerosol activation: 1. Single aerosol type, J. Geophys. Res., 103, 6123-6131, 1998.

Barahona, D. and Nenes, A.: Parameterization of cloud droplet formation in large-scale models: Including effects of entrainment, J. Geophys. Res., 112, D16206, doi:10.1029/2007JD008473, 2007.

Barahona, D., West, R. E. L., Stier, P., Romakkaniemi, S., Kokkola, H., and Nenes, A.: Comprehensively accounting for the effect of giant $\mathrm{CCN}$ in cloud activation parameterizations, Atmos. Chem. Phys., 10, 2467-2473, doi:10.5194/acp-10-2467-2010, 2010.

Chuang, P. Y., Charlson, R. J., and Seinfeld, J. H.: Kinetic limitations on droplet formation in clouds, Nature, 390, 594-596, 1997.

Feingold, G. and Heymsfield, A.: Parameterizations of condensational growth of droplets for use in general circulation models, J. Atmos. Sci., 49, 2325-2342, 1992.

Fountoukis, C. and Nenes, A.: Continued development of a cloud droplet formation parameterization for global climate models, J. Geophys. Res., 110, D11212, doi:10.1029/2004JD005591, 2005.

Fountoukis, C., Nenes, A., Meskhidze, N., Bahreini, R., Conant, W. C., Jonsson, H., Murphy, S., Sorooshian, A., Varutbangkul, V., Brechtel, F., Flagan, R. C., and Seinfeld, J. H.: Aerosol cloud drop concentration closure for clouds sampled during the International Consortium for Atmospheric Research on Transport and Transformation 2004 campaign, J. Geophys. Res., 112, D10S30, doi:10.1029/2006JD007272, 2007.

Ghan, S., Chuang, C., and Penner, J.: A parameterization of cloud droplet nucleation. Part I: Single aerosol type, Atmos. Res., 30, 198-221, doi:10.1016/0169-8095(93)90024-I, 1993.

Ghan, S., Guzman, G., and Abdul-Razzak, H.: Competition between sea salt and sulfate particles as cloud condensation nuclei, J. Atmos. Sci., 55, 3340-3347, 1998.

Ghan, S., Abdul-Razzak, H., Nenes, A., Ming, Y., Liu, X., Ovchinnikov, M., Meskhidze, N., Xu, J., and Shi, X.: Droplet nucleation: physically-based parameterization and comparative evaluation, J. Adv. Model. Earth. Syst., 3, D10S30, doi:10.1029/2011MS000074, 2011.

Intergovernmental Panel on Climate Change, .: Fourth Assessment Report: Climate Change 2007: Working Group I Report: The Physical Science Basis, Geneva, IPCC 2007.
Kumar, P., Sokolik, I. N., and Nenes, A.: Parameterization of cloud droplet formation for global and regional models: including adsorption activation from insoluble CCN, Atmos. Chem. Phys., 9, 2517-2532, doi:10.5194/acp-9-2517-2009, 2009.

Lamarque, J.-F., Bond, T. C., Eyring, V., Granier, C., Heil, A., Klimont, Z., Lee, D., Liousse, C., Mieville, A., Owen, B., Schultz, M. G., Shindell, D., Smith, S. J., Stehfest, E., Van Aardenne, J., Cooper, O. R., Kainuma, M., Mahowald, N., McConnell, J. R., Naik, V., Riahi, K., and van Vuuren, D. P.: Historical (1850-2000) gridded anthropogenic and biomass burning emissions of reactive gases and aerosols: methodology and application, Atmos. Chem. Phys., 10, 7017-7039, doi:10.5194/acp10-7017-2010, 2010.

Lathem, T. L., Kumar, P., Nenes, A., Dufek, J., Sokolik, I. N., Trail, M., and Russel, A.: Hygroscopic properties of volcanic ash, Geophys. Res. Lett., 38, L11802, doi:10.1029/2011GL047298, 2011.

Liu, X., Easter, R. C., Ghan, S. J., Zaveri, R., Rasch, P., Shi, X., Lamarque, J.-F., Gettelman, A., Morrison, H., Vitt, F., Conley, A., Park, S., Neale, R., Hannay, C., Ekman, A. M. L., Hess, P., Mahowald, N., Collins, W., Iacono, M. J., Bretherton, C. S., Flanner, M. G., and Mitchell, D.: Toward a minimal representation of aerosols in climate models: description and evaluation in the Community Atmosphere Model CAM5, Geosci. Model Dev., 5, 709-739, doi:10.5194/gmd-5-709-2012, 2012.

Meskhidze, N., Nenes, A., Conant, W. C., and Seinfeld, J. H.: Evaluation of a new cloud droplet activation parameterization with in situ data from CRYSTAL-FACE and CSTRIPE, J. Geophys. Res., 110, D16202, doi:10.1029/2004JD005703, 2005.

Ming, Y., Ramaswamy, V., Donner, L. J., and Phillips, V. T. J.: A new parameterization of cloud droplet activation applicable to general circulation models, J. Atmos. Sci., 63, 1348-1356, 2006.

Morales Betancourt, R. and Nenes, A.: Understanding the contributions of aerosol properties and parameterization discrepancies to droplet number variability in a global climate model, Atmos. Chem. Phys., 14, 4809-4826, doi:10.5194/acp-14-48092014, 2014.

Nenes, A. and Seinfeld, J. H.: Parameterization of cloud droplet formation in global climate models, J. Geophys. Res., 108, 4415, doi:10.1029/2002JD002911, 2003.

Nenes, A., Ghan, S., Abdul-Razzak, H., Chuang, P. Y., and Seinfeld, J. H.: Kinetic limitations on cloud droplet formation and impact on cloud albedo, Tellus, 53B, 133-149, 2001.

Petters, M. D. and Kreidenweis, S. M.: A single parameter representation of hygroscopic growth and cloud condensation nucleus activity, Atmos. Chem. Phys., 7, 1961-1971, doi:10.5194/acp-71961-2007, 2007.

Pinsky, M., Khain, A., Mazin, I., and Korolev, A.: Analytical estimation of droplet concentration at cloud base, J. Geophys. Res., 117, D18211, doi:10.1029/2012JD017753, 2012.

Pruppacher, H. and Klett, J.: Microphysics of clouds and precipitation, Atmospheric and oceanographic sciences library, Kluwer Academic Publishers, 2nd rev, 1997.

Seinfeld, J. and Pandis, S.: Atmospheric chemistry and physics: from air pollution to climate change, A Wiley-Intersciencie publications, Wiley, 2006.

Shipway, B. and Abel, S.: Analytical estimation of cloud droplet nucleation based on an underlying aerosol population, Atmos. Res., 96, 344-355, doi:10.1016/j.atmosres.2009.10.005, 2010. 
Sorjamaa, R. and Laaksonen, A.: The effect of $\mathrm{H}_{2} \mathrm{O}$ adsorption on cloud drop activation of insoluble particles: a theoretical framework, Atmos. Chem. Phys., 7, 6175-6180, doi:10.5194/acp-76175-2007, 2007.
Twomey, S.: The nuclei of natural cloud formation. Part II: The supersaturation in natural clouds and the variation of cloud droplet concentration, Geof. Pura Appl., 43, 243-249, 1959. 